\title{
Investigating the performance of seals using photoelastic experimental hybrid method and finite element analysis
}

\author{
Bruno R. MOSE*, Dong-Kil SHIN* and Dong-Chul SHIN** \\ *School of Mechanical Engineering, Yeungnam University, 280, Daehak-Ro, Gyeongsan, Gyeongbuk 38541, Korea \\ ${ }^{* *}$ Department of Mechanical Engineering, Koje College, Geoje, Gyeongnam, 53325, Korea \\ E-mail: dkshin@yu.ac.kr
}

Received: 24 January 2017; Revised: 23 March 2017; Accepted: 21 April 2017

\begin{abstract}
In this paper, performance behavior of seals under compression and various hydraulic pressures were investigated. Two approaches (experimental and numerical) have been used. It was found that significant improvements in seal performance can be achieved by carefully combining rectangular and circular profiles into one seal. The folding mechanism at the step of the seal with $\mathrm{H}_{1} / \mathrm{H}_{2}=2.5$ was found to contribute the seal's best performance. Further, for each loading condition, contact stresses were greater than the applied hydraulic pressures. This important sealing criterion confirmed that the new seal geometry has good sealing capability. Contrary to previous studies, contact stresses were found to possess a non Hertzian profile. By measuring contact lengths using a video microscope, the seal with new geometry recorded higher contact lengths compared with seals having circular profile. The fringe patterns and deformation behavior from numerical models showed remarkable similarity with experimental results.
\end{abstract}

Key words : Seals, Phoelaticity, PEHM, Hydraulic pressures, Contact stresses, FEA

\section{Introduction}

Seals are important leakage control devices found in many mechanical components such as the rocket propulsion parts, aircraft parts, and other machineries because of their ability to prevent leakage of fluids into sealed compartments. Seals also provide an important mechanism against the entrance of external contaminating substances such as pollutants, powders, gases, and so on. A number of cross sectional shapes for seals are available. These include circular-, rectangular-, and triangular- profiles. Seals with circular shapes have been widely used because of their advantages which include low cost and simple mounting requirements (Lee et. al., 2008). However, seals with circular profiles have been reported to provide unsatisfactory performance due to their tendency to twist during use (Lee et al., 2008, Shin et al., 2014). This unfavourable twisting behaviour is commonly known as spiral failure (Parker, 2008).

Due to increased technological advancement, seals are required to operate under severe conditions such as those in aircraft and rocket propulsion parts without failure. Any failure of these components can lead to significant damage of mechanical systems and excessive leakage of fluid to the environment. In recent years, considerable effort has been devoted to study seals in order to provide an in-depth understanding of the behaviour of seals with a view to assure their safe usage during operation.

During installation and operation, seals are pre-stressed to about $20 \%$ squeeze and pressurized with high fluid pressures. This causes significant stresses to be produced at the contact surfaces of seals. The resulting stresses from assembly and pressurization can degrade the performance and operating lifespan of seals as well as their reliability. White and Denny (1947) noted that seal wear occurs mainly in regions of high tensile strains leading to high abrasion and eventual fracture of seals. To minimize failure and enhance seal performance, a stress evaluation is the first step for failure analysis. A number of investigators have studied the stresses produced in seals. Kim et al. (2009) used specimens prepared from epoxy to study stresses in a seal subjected to a vertical compression and lateral pressures of up to 3.92 $\mathrm{MPa}$. The authors observed that the contact stresses followed the Hertzian profile with a non-zero stress value at the 
boundary. Johannesson and Kassfeld (1989) measured the contact stress distributions of an elastomeric seal. Contact stresses in seals have also been studied by Strozzi (1986) using photoelastic experiments. Photoelaticity is an attractive experimental method because of its ability to give whole field observation of stresses for complicated shapes and loading conditions. Medri and Strozzi (1984) conducted photoelastic experiments, and noted that photoelasticity is a useful technique that can be used to assess the accuracy of numerical results. Bignardi et al. (1999) used both isoclinic and isochromatic data from photoelasticity to describe stress fields and contact pressures of lip seals.

The use of isoclinic and isochromatic fringes has however, been faced with technical difficulties. For instance, it is not easy to differentiate between isoclinic and isochromatic fringe lines. Moreover, isoclinic fringes are time consuming to obtain (Medri and Strozzi, 1984) and tend to be less reliable. To overcome the difficulties associated with isoclinic fringes in stress analysis, hybrid methods have been suggested (Lin and Rowlands, 1999). These methods combine advantages of experimental, numerical and analytical methods. Photoelastic experimental hybrid method (PEHM) is one of the hybrid methods that has been applied to analyze stresses in structures with considerable success. One of the merits of PEHM is its ability to separate stress components using isochromatic fringes only without using isoclinic fringes. Nam et al. (2011) used PEHM to obtain contact stresses of O-ring seals. Hawong et al. (2009, 2010) employed PEHM to study the interior and contact stresses of seals with circular cross sections.

As noted, many studies have been carried out with the aim of evaluating the stresses produced in seals with circular (Kim et al., 2007, Hawong et al., 2009, 2010,Nam et al., 2011) and rectangular (Johannesson and Kassfeld, 1989, Strozzi, 1986) cross sections. There is little information in the open literature on the performance behavior of seals with the geometry that combines rectangular and circular profiles. Further, there are contradictory reports regarding the profile of contact stress fields. It is generally reported (Kim et al., 2007) that the contact stress field has the Hertzian profile when the circular-shaped seal is under compression only, without lateral pressure. Karaszkiewicz (1990) and Johannesson (1978) noted that even with both compression and medium pressure, the contact stresses have a Hertzian profile. These observations need to be investigated through further studies.

The main objective of this paper is to assess the performance behaviour of a contact seal with a new geometry of combined rectangular and circular profiles to contribute to better designs of seals and seal systems. This is especially important for various reasons. Firstly, the presence of tensile stresses in the seal may result in cracking and hence failure. Secondly, to prevent leakages, the static contact pressure must exhibit a larger value than the hydraulic pressure under operational conditions. Both experimental and numerical methods were used in this work. Experimental work is particularly useful not only for direct application of results in industry but also as a powerful tool to verify theoretical and numerical predictions.

To achieve the objectives, photoelastic experiments were performed under various loading conditions to obtain isochromatic fringes of the stress fields. An image processing software, Image Pro Plus was then used to analyze isochromatic fringes from photoelastic experiment. The data from the image processing software was used in a computer program to determine the interior and contact stresses of seals with different cross sectional geometries. The profile of the contact stresses was obtained and was shown to have non-Hertzian profile. The extrusion behaviour and contact lengths at typical seal working pressures were determined. The contact lengths were measured using a video microscope.

Hyperelastic properties of epoxy material used to make photoelastic models were measured. A numerical method was then used to investigate the seal behaviour at a pre-load of $20 \%$ and various hydraulic pressures using Abaqus. The numerical results were correlated with experimental results and remarkable similarity was observed between experimental and numerical results.

\section{Measurement of internal stress by photoelastic experimental hybrid method (PEHM) 2.1 Seal specimen}

Due to spiral failure resulting from circular shaped seals (Fig. 1.a), a new seal profile was proposed (Fig. 1.b). The seal was designed to possess a flat base and can be a suitable replacement to mechanical seals with circular cross section due to its better resistance to spiral failure (Parker, 2008). The seal possesses an additional advantage of fitting easily into rectangular grooves commonly used in industry. Furthermore, the seal combines the advantages of both circular and rectangular shapes which makes it more attractive than individual use of either the circular or rectangular seal. 


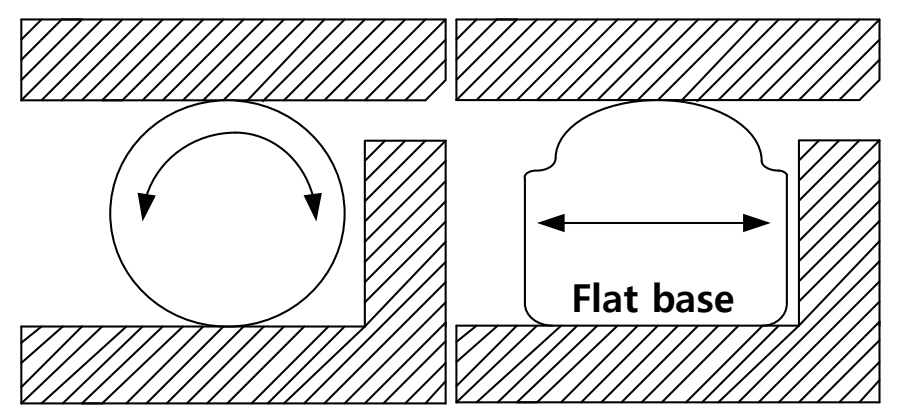

(a)

(b)

Fig. 1. Motion of seal ring. (a) Twisting action of circular shaped seals and (b) resistance to twisting of D-ring

To investigate performance behavior of the new seal, specimens (Fig. 2 (a)) were first fabricated from aluminium metal having a cross sectional height of $7 \mathrm{~mm}$. The cross sectional height, $\mathrm{H}$ was the sum of $\mathrm{H}_{1}$ and $\mathrm{H}_{2}$. The ratio of $\mathrm{H}_{1} / \mathrm{H}_{2}$ was varied between 1 and 3.5 as shown in Fig 2 (b) for the case of $\mathrm{H}_{1} / \mathrm{H}_{2}=3.5$. The metallic seals served as patterns to make pink silicone molds. Using the molds, photoelastic models were cast from a mixture of Araldite and hardener manufactured by Hutsman. Details of the casting procedure are provided in (Nam et al., 2008). The fabricated seal model, geometric dimensions and co-ordinate system used in seal analysis are shown in Fig. 2. From the fabricated model, it is noted inner diameter was $121.5 \mathrm{~mm}$. In the co-ordinate system (Fig. 2 (c)), $X Y$ is global coordinate and $x y$ local coordinate. On the front region, $x_{f}=Y$ and $y_{f}=-X$ while on the upper side, $x_{u}=-X$ and $y_{u}=-Y$.

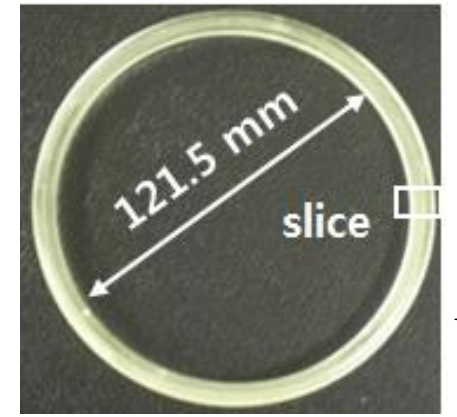

(a)

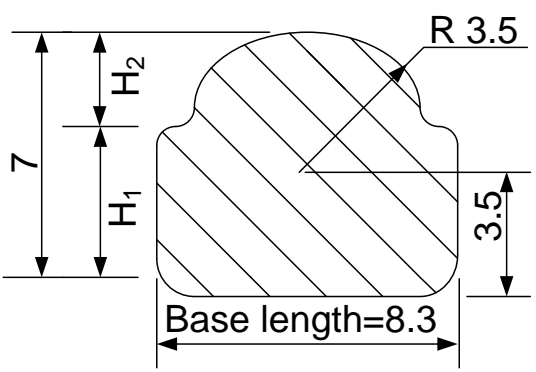

(b)

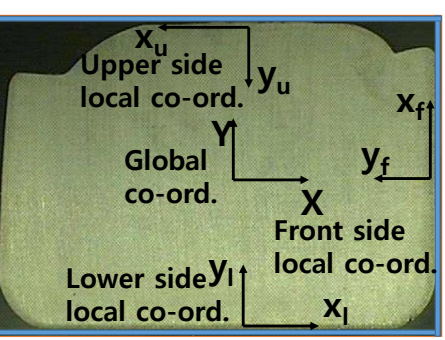

(c)

Fig. 2. Specimen: (a) fabricated model, (b) geometric dimensions of cross section, and (c) co-ordinate system of seal ring

\subsection{Loading equipment}

The test equipment that was used in PEHM is shown in Fig. 3. The equipment consists of housing cylinder and seal ring guide. The equipment is designed to give $20 \%$ squeeze and allow application of hydraulic pressures to the seals. Loading directions and sectional view are shown in Fig. 3(b). Details on the working principle of the test equipment are provided in reference (Hawong et al., 2010). 


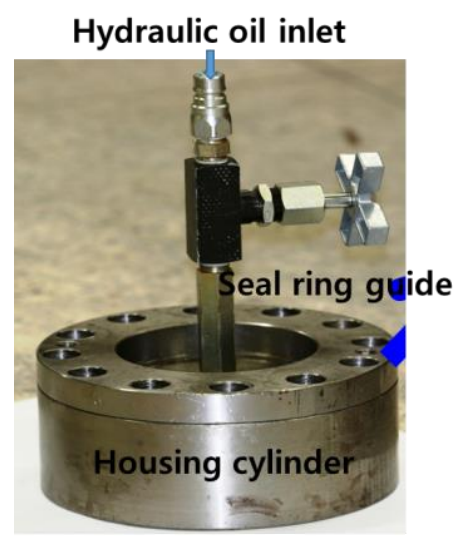

(a)

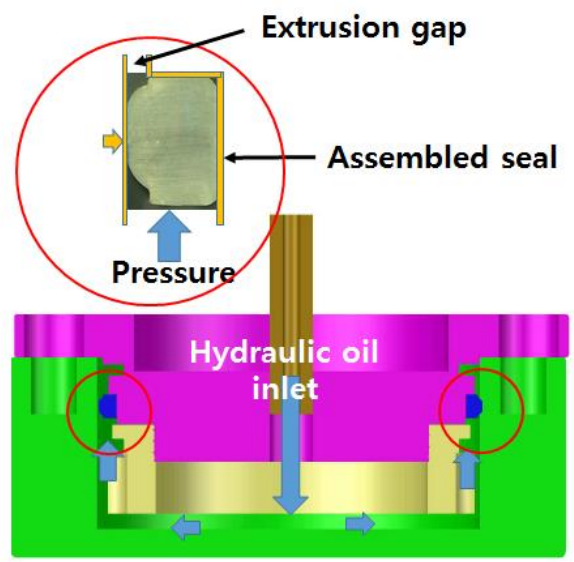

(b)

Fig.3. (a) Loading equipment designed to give $20 \%$ squeeze and various hydraulic pressures, and (b) sectional view of loading device with assembled seal

\subsection{Stress freezing and PEHM}

The seal model was installed into a specially designed loading equipment (Fig. 3(a)) and stress freezing was performed to lock stress fringes by heating seal models above glass transition temperature according to the cycle in (Nam et al., 2008). During stress freezing cycle, hydraulic pressure was applied through the hydraulic oil inlet shown in Fig. 3 (b). Small slices (Fig. 2 (a)) were cut out from stress frozen seals and polished to a thickness of about 1 mm. Polished slices were mounted on the transparent photoelastic experimental device Mod. 061-17 and their isochromatic fringes were recorded using a digital camera. From isochromatic fringes, image analysis was done using imaging software, Image Pro Plus (IPP). The most bright and dark points in the black (x.0 order) and white bands (x.5 order) of isochromatics were found by using the "Image Pro Plus". And then, the fringe data in PEHM was obtained after adding the fringe orders at the points in the region of stress analysis. The data obtained from IPP was combined with geometric and loading condition of the seal to evaluate stresses using the formula in eq. (1). A detailed derivation of eq. (1) can be obtained from (Hawong et al., 2006).

$$
\begin{aligned}
& \sigma_{x}(Z)=\sum_{n=1}^{N} \operatorname{Re}\left\{C_{n}[2 F(n, z)-G(n, z)]+\bar{C}_{n} F(n, z)\right\} \\
& \sigma_{y}(Z)=\sum_{n=1}^{N} \operatorname{Re}\left\{C_{n}[2 F(n, z)+G(n, z)]-\bar{C}_{n} F(n, z)\right\} \\
& \tau_{x y}(Z)=\sum_{n=1}^{N} \operatorname{Im}\left\{C_{n} G(n, z)-\bar{C}_{n} F(n, z)\right\}
\end{aligned}
$$

where; $F(n, z)=\frac{n}{2} z^{\frac{n}{2}-1}, G(n, z)=\frac{n}{2}\left[\left(\frac{n}{2}-1\right) \bar{z}-\frac{n}{2} z\right] z^{\frac{n}{2}-2}$

In eq. (1), $\mathrm{z}$ is a complex variable given by the expression $\mathrm{z}=\mathrm{x}+\mathrm{iy} ; \mathrm{i}=\sqrt{-1}, \operatorname{Re}$ and $\operatorname{Im}$ are the real and imaginary parts of the complex function. The value of $C_{n}=a_{n}+i b_{n}$ was obtained through computational method. Overbar of $C_{n}$ in eq. (1) means the conjugate of $C_{n}$. The number of isochromatic fringes is denoted by $\mathrm{N}$ and is easily obtained from stress-optic law in eq. (2). The stress optic law is a useful equation that gives the relationship between the observed isochromatic fringes and the stresses. It serves as an important tool in converting fringe data into stress distributions. The procedure of using experimental data with numerical computation to determine $C_{n}$ and substituting it in eq. (1) to get stress components $\left(\sigma_{x}, \sigma_{y}, \tau_{x y}\right)$ is known as PEHM. The state of stress in the seal structure was known when the regenerated graphic fringes were similar to the actual experimental fringes. When regenerated fringes were not similar to actual ones, the process was repeated until the actual and regenerated fringe patterns showed good fitting. 
Stress analysis of the seal was performed independently for the upper and front sides using the coordinate system described in Fig. 2 (c).

$$
\left(\frac{f_{\sigma} \cdot N}{t}\right)^{2}=\left(\sigma_{x}-\sigma_{y}\right)^{2}+\left(2 \tau_{x y}\right)^{2}
$$

In eq. (2), $f_{\sigma}$ is the stress fringe value, $\mathrm{N}$ is the fringe order, and $\mathrm{t}$ is the thickness of the specimen. The stress fringe value, $\mathrm{f}_{\sigma}$, of the photoelastic material used in this study was $242.6 \mathrm{~N} / \mathrm{m}$ (Shin et al., 2014).

\section{Results of photoelastic experimental hybrid method (PEHM) 3.1 Isochromatic fringes}

The isochromatic fringe patterns from stress frozen seal models with various ratios of $\mathrm{H}_{1}$ to $\mathrm{H}_{2}$ and compressed to $20 \%$ squeeze as hydraulic pressure was varied are shown in Fig. 4. The fringes obtained from O-ring seal have been included for comparison (Nam et al., 2011). It was observed that when hydraulic pressure increased, the fringe order increased with the region near the extrusion gap recording the highest fringe order. The patterns of the fringe lines at the side where pressure was applied were almost vertical for each loading condition regardless of the cross sectional profile and ratio $\mathrm{H}_{1} / \mathrm{H}_{2}$. At $3.92 \mathrm{MPa}$ hydraulic pressure, all the seals recorded higher fringe orders compared to those at lower pressures. From this study, seal material started being forced out through the narrow gap of the groove when hydraulic pressure was 5.4 MPa for the seal with $\mathrm{H}_{1} / \mathrm{H}_{2}=3.5$. The forcing out phenomenon of seal material through the gap is commonly described as extrusion. To initiate extrusion in the seal with $\mathrm{H}_{1} / \mathrm{H}_{2}=2.5$, a pressure of $6.5 \mathrm{MPa}$ was required. For the seal with circular cross section, extrusion had already occurred at $3.92 \mathrm{MPa}$. Therefore, the pressures required to initiate extrusion were dependent on the geometric condition of the seal.

To determine the geometric effect of $\mathrm{H}_{1}$ and $\mathrm{H}_{2}$ on internal stresses, a detailed analysis of the seals was done using PEHM. Firstly, data along the fringe lines of the actual isochromatics recorded by a digital camera was collected using Image Pro Plus, an image processing software. During data collection, the seal had to be rotated accordingly using the co-ordinate system in Fig. 2 (c). Collected data by Image Pro Plus was combined with geometric and loading conditions and transferred into a computer based program that was developed by the authors for stress analysis. The state of stress in the seal structure was determined when the actual and regenerated isochromatics were similar to each other.

In Fig. 5, the actual and regenerated isochromatics of the upper and front sides of the seal under $20 \%$ squeeze and hydraulic pressure of $3.92 \mathrm{MPa}$ are described. On the other hand, Fig. 6 shows the actual and regenerated isochromatics of the upper and front sides of the seal ring with a fractional compression of $20 \%$ and 5.4 MPa hydraulic pressure. In both cases, it was observed that the actual isochromatics in the region highlighted by the rectangle were very similar to the regenerated isochromatics; with cross marks "+" on regenerated isochromatics located along the centre lines of the isochromatic bands. It is to noted that the regenerated isochromatics have been rotated to correspond to the co-ordinate system described in Fig. 2 (c). The $x / \mathrm{a}$ and $y / \mathrm{a}$ on regenerated isochromatics represent the $x$ and $y$ distances which have been normalized by half the contact length, a. 

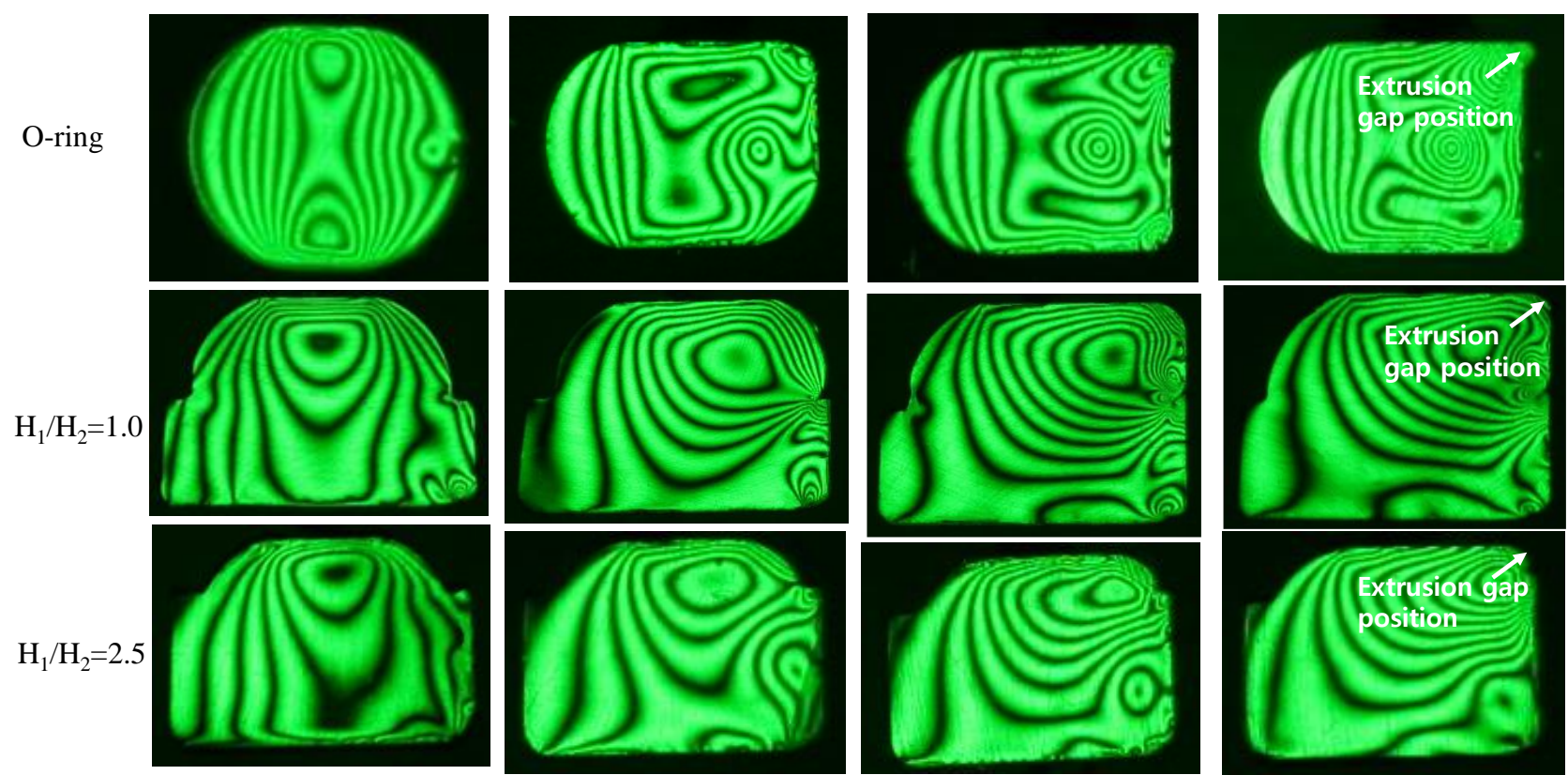

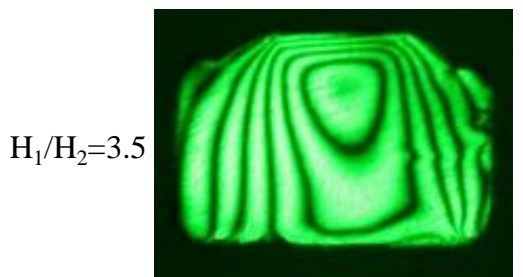

(a) $0 \mathrm{MPa}$

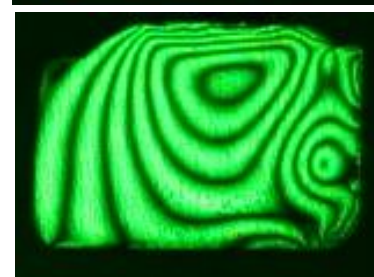

(b) $0.98 \mathrm{MPa}$

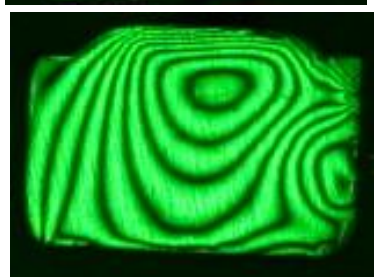

(c) $2.94 \mathrm{MPa}$

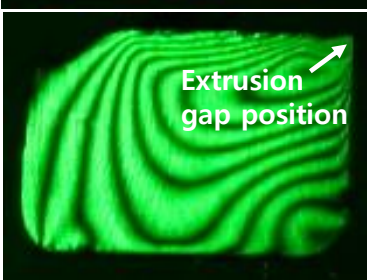

(d) $3.92 \mathrm{MPa}$

Fig. 4. Comparison of isochromatic fringe patterns according to ratios $\mathrm{H}_{1} / \mathrm{H}_{2}$ and hydraulic pressure

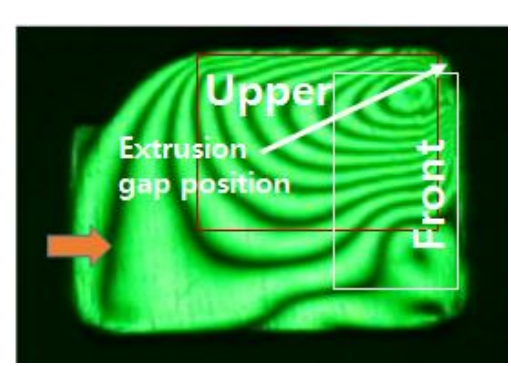

(a) Actual

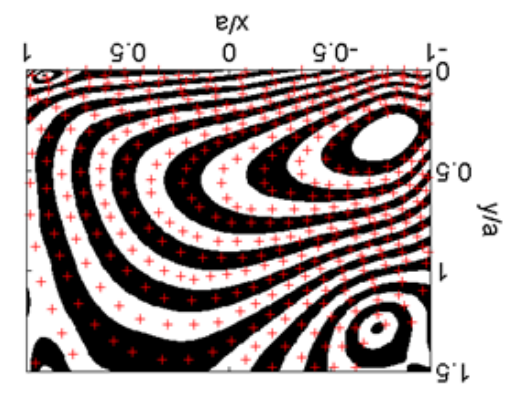

(b) Regenerated, U: a=3.03 mm

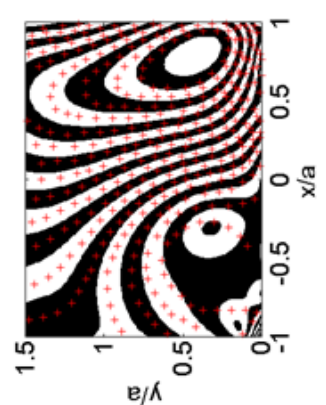

(c) Regenerated, F: $\mathrm{a}=2.745 \mathrm{~mm}$

Fig. 5. Actual and regenerated isochromatics of upper and front side of seal with $\mathrm{H}_{1} / \mathrm{H}_{2}=2.5$ (hydraulic pressure: 3.92 $\mathrm{MPa}, \mathrm{U}$ : upper side, F: front side)

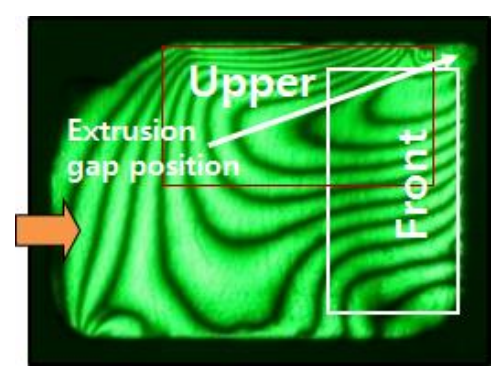

(a) Actual

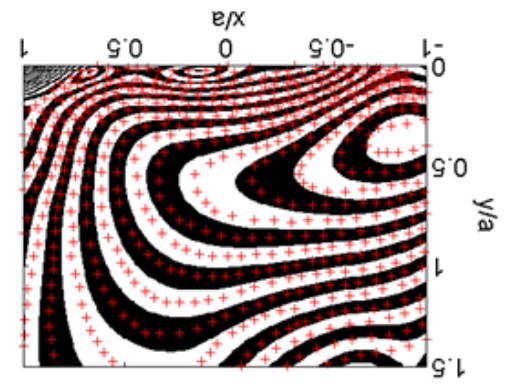

(b) Regenerated, U: a= $3.686 \mathrm{~mm}$

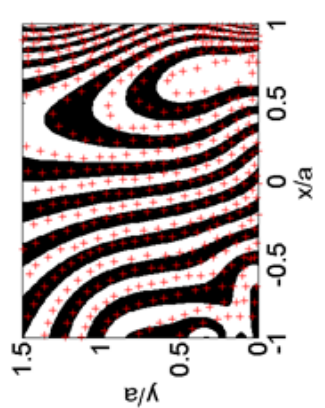

(c) Regenerated, F: $\mathrm{a}=2.28 \mathrm{~mm}$

Fig. 6. Actual and regenerated isochromatics of upper and front side of seal with $\mathrm{H}_{1} / \mathrm{H}_{2}=3.5$ (hydraulic pressure: $5.4 \mathrm{MPa}$, U: upper side, F: front side) 


\subsection{Analysis of internal and contact stresses using PEHM}

With similarity between regenerated and actual experimental fringes, the stress components $\sigma_{x}, \sigma_{y}, \tau_{x y}$ and von Mises stresses, $\sigma_{\mathrm{vm}}$, in the interior of the seal structure were determined. For brevity, only the interior stresses on the upper side of the seal with $\mathrm{H}_{1} / \mathrm{H}_{2}=3.5$ obtained from Fig 6 are shown in Fig. 7. From the stress contours in Fig. 7, the highest magnitudes of $\sigma_{x}$ and $\sigma_{y}$ were $10.4 \mathrm{MPa}$ and 10.9 $\mathrm{MPa}$ while those of $\tau_{x y}$ and $\sigma_{\mathrm{vm}}$ were $0.32 \mathrm{MPa}$ and $10.6 \mathrm{MPa}$ respectively. It is evident that the magnitudes of $\sigma_{y}$ were slightly higher than those of $\sigma_{x}$ and the values of $\tau_{x y}$ were generally very small. It is further noted that the highest magnitudes of stresses generally occurred in the region close to the extrusion gap located at the bottom left corner of the stress contours.

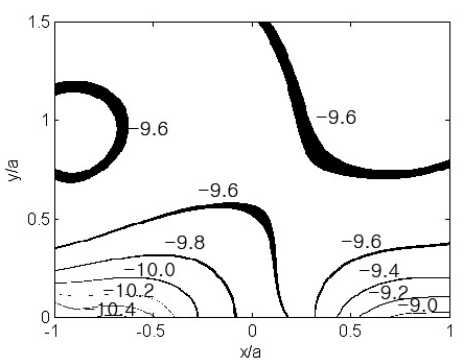

(a) $\sigma_{\mathrm{x}}$

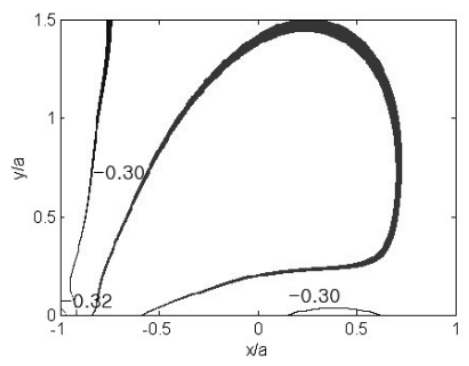

(c) $\tau_{\mathrm{xy}}$

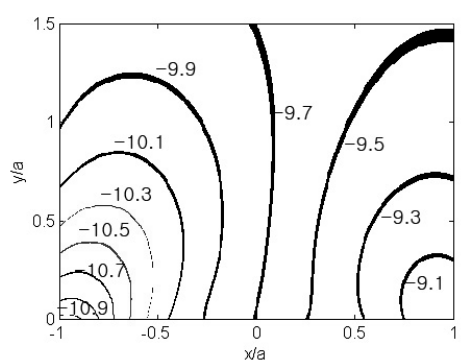

(b) $\sigma_{\mathrm{y}}$

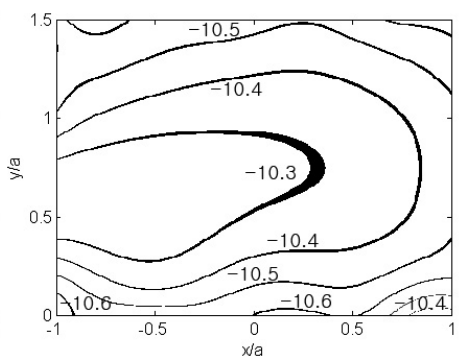

(d) $\sigma_{\mathrm{vm}}$

Fig. 7. Stress contours of upper side of the seal with $\mathrm{H}_{1} / \mathrm{H}_{2}=3.5$ drawn in local $x-y$ co-ordinate (applied pressure: $5.4 \mathrm{MPa}$, $\mathrm{a}=3.686 \mathrm{~mm}$ )

In a similar manner described above, the internal stresses of the seals with various ratios of $\mathrm{H}_{1}$ to $\mathrm{H}_{2}$ were analyzed using PEHM. From the analysis, the highest/greatest shear stress component, $\tau_{\mathrm{xy}}$ close to the extrusion gap was obtained and the results are summarized in Fig. 8. It was observed that among the seals investigated in this research, the seal with $\mathrm{H}_{1} / \mathrm{H}_{2}=2.5$ experienced the lowest value of shear stresses, $\tau_{\mathrm{xy}}$ at all loading conditions. Moreover, the stress components $\sigma_{\mathrm{x}}$ and $\sigma_{\mathrm{y}}$ for the seal with $\mathrm{H}_{1} / \mathrm{H}_{2}=2.5$ were generally lower compared to other seals under the same loading condition of compression and hydraulic pressure. This may be attributed to the folding mechanism that was observed at the step near the extrusion gap. The folding mechanism implies that the entangled polymer chains got disentangled. This disentanglement weakens the interactions between epoxy molecules. This in turn lowers the stresses compared to other seals where folding mechanism was not observed.

On the basis of maximum shear criterion (see eq. (3)), the seal with a ratio of $\mathrm{H}_{1} / \mathrm{H}_{2}=2.5$ demonstrates better resistance to shear. The shear stress is considered in engineering stress models as the most damaging as it controls crack initiation. The seal with $\mathrm{H}_{1} / \mathrm{H}_{2}=2.5$ registered the lowest magnitude of $\tau_{\mathrm{xy}}$ which when substituted in eq. (3) together with lower values of $\sigma_{x}$ and $\sigma_{y}$ for the seal with $\mathrm{H}_{1} / \mathrm{H}_{2}=2.5$ will yield lowest $\tau_{\max }$. Since seals fail due to fracture at the region near the extrusion gap (White and Denny, 1947, Medri and Strozzi, 1984), it can reasonable to suggest that the seal with $\mathrm{H}_{1} / \mathrm{H}_{2}=2.5$ can resist fracture and hence failure. Therefore, seals with the proposed profile can serve as alternative seals for high pressure applications; particularly in areas where performance of circular shaped seals is unsatisfactory.

$$
\left(2 \tau_{\max }\right)^{2}=\left(\sigma_{x}-\sigma_{y}\right)^{2}+\left(2 \tau_{x y}\right)^{2}=\left(\frac{f_{\sigma} \cdot N}{t}\right)^{2}
$$




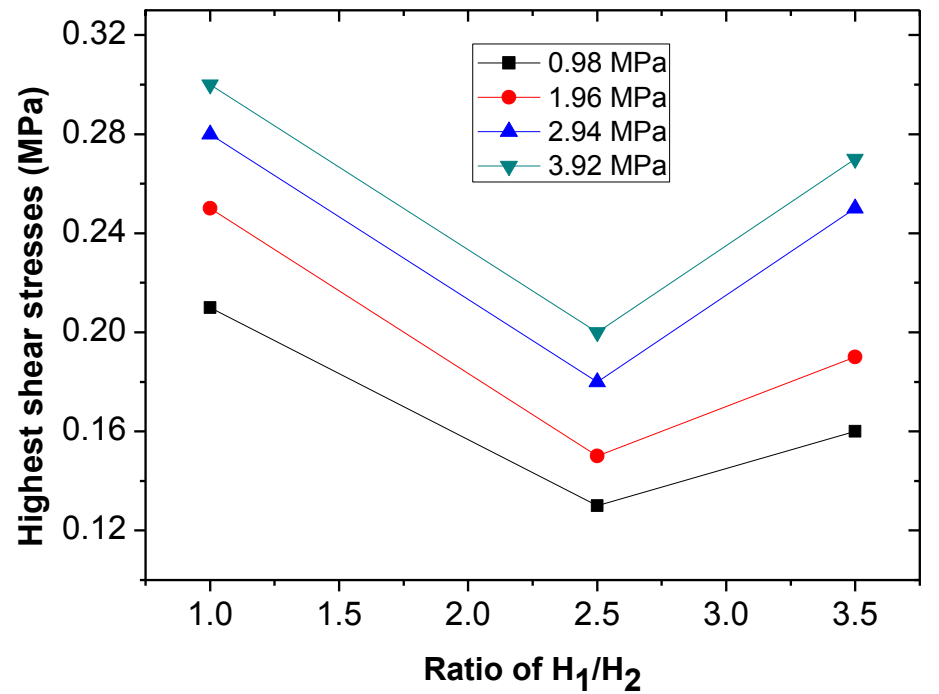

Fig. 8. Comparison of highest $\tau_{x y}$ at the region close to extrusion gap for D-rings with various ratios of $\mathrm{H}_{1} / \mathrm{H}_{2}$

The sealing capability depends greatly upon the contact stresses between the seal and the surfaces with which it comes into contact (Green and English, 1994). It is generally known that leakages will occur when the pressure differential across the seal exceeds the contact stresses. In this study, a detailed analysis of the contact stresses was carried out on the upper side of the seal models to establish whether the sealing condition to avoid leakage was met. The selection of the upper side was informed by the high intensity of stresses experienced in this region as evidenced by the high order isochromatic fringes (see Fig. 4). Representative results of this analysis are shown in Figs. 9. It is observed that the contact stresses were greater than the applied pressures for each of the loading conditions. It is also observed that the contact stresses followed a non-Hertzian profile and were almost constant over the contact length. The performance of the seal with the proposed shape was found to be satisfactory in avoiding leakages since the contact stresses were higher than applied pressure. Therefore this seal shape offers new possibilities as an alternative to the circular shaped seal.

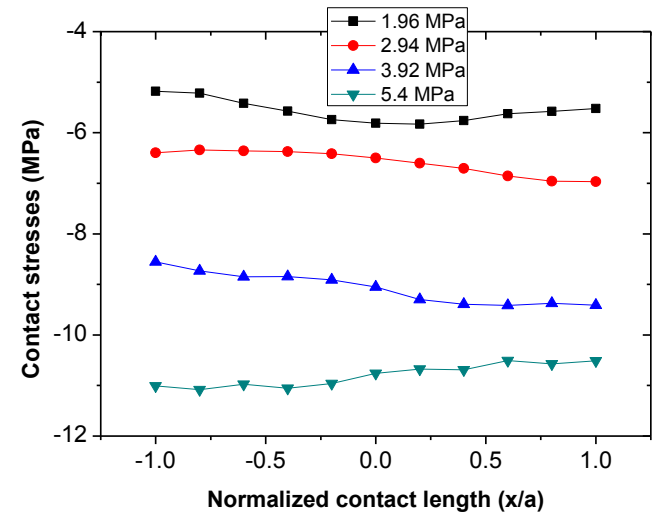

(a)

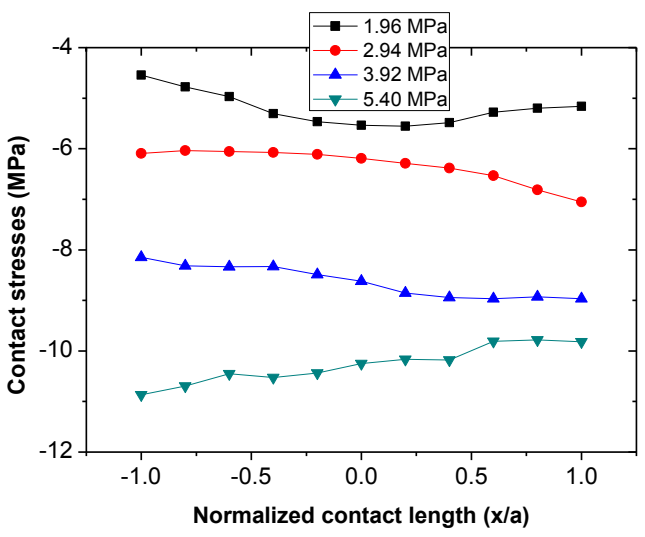

(b)

Fig. 9. Variation of contact stresses at upper side of contact region with respect to hydraulic pressure (a) $\mathrm{H}_{1} / \mathrm{H}_{2}=2.5$ and (b) $\mathrm{H}_{1} / \mathrm{H}_{2}=3.5$

\subsection{Contact length}

The contact lengths of seals play a significant role in determining the packing ability of sealing elements. In this study, a video microscope was used to measure the contact lengths of the seals as the hydraulic pressure increased. The results are shown in Fig. 10 for the case of $\mathrm{H}_{1} / \mathrm{H}_{2}=3.5$. The results indicated that the contact lengths increased from 4.26 
$\mathrm{mm}$ to $4.77 \mathrm{~mm}$ as the hydraulic pressure increased from $0.98 \mathrm{MPa}$ to $3.92 \mathrm{MPa}$.

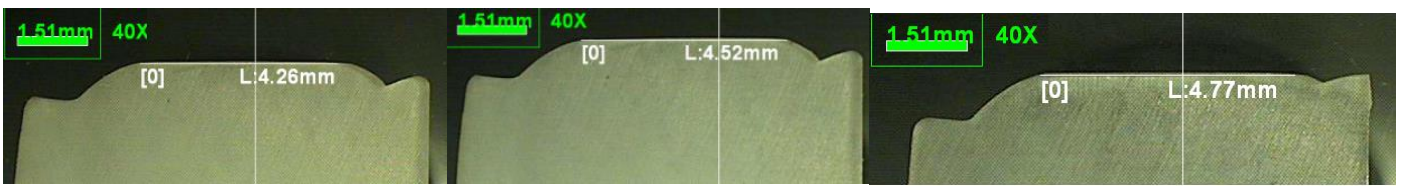
(a) $0.98 \mathrm{MPa}$
(b) $2.94 \mathrm{MPa}$
(c) $3.92 \mathrm{MPa}$

Fig. 10. Variation of contact lengths with hydraulic pressure for upper part of seal $\left(\mathrm{H}_{1} / \mathrm{H}_{2}=3.5\right.$, magnification: $\left.\mathrm{x} 40\right)$

The results of packing ability of the seals with combined circular and rectangular profiles from this study were compared with those of the circular shaped seal in (Hawong et al., 2010). The results are shown in Fig. 11. The contact lengths of seals with the suggested profile were similar to each other at all hydraulic pressures. Interestingly, at zero pressure, the contact lengths for all the seals including the O-ring were similar. However, at 3.92 MPa, the contact lengths of the proposed seals were higher than those of the O-ring seal. Thus, the packing ability of these seals becomes even better than that of the circular shaped seal especially at high fluid pressures.

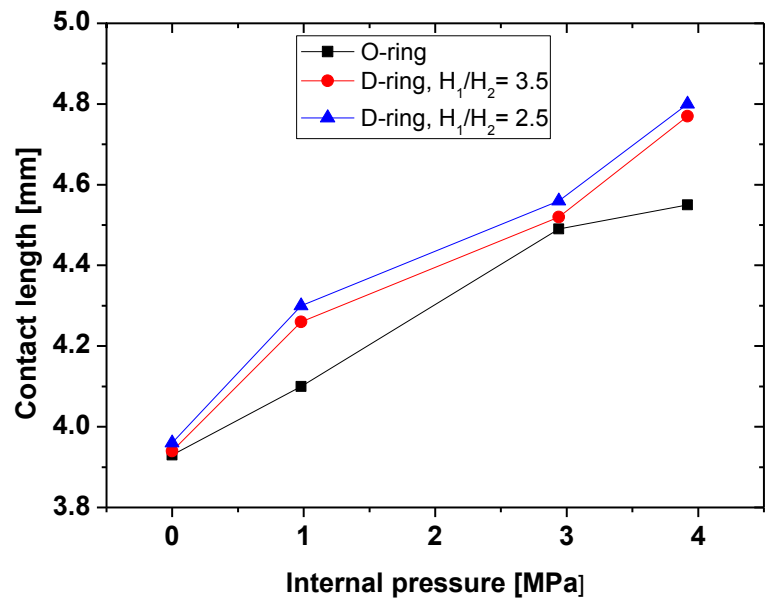

Fig. 11. Comparison of contact lengths of D-shaped and circular shaped seals under $20 \%$ compression ratio and various hydraulic pressures

\section{Analysis of the deformation behavior by Finite Element Analysis}

Deformation behavior of the D-shaped seal was investigated by finite element analysis (Abaqus, Dassault systemes). Fig. 12 shows the finite element model of the sealing system with $\mathrm{H}_{1} / \mathrm{H}_{2}=2.5$. Considering the axisymmetric deformation about centre axis of the seal-ring and long length of $4.15 \mathrm{~mm}$ compared with the width $3.5 \mathrm{~mm}$, two dimensional analysis was performed. Plane stress element with full integration (CPS4) was used, the number of elements was 6233, and the size of mesh was about $0.1 \mathrm{~mm}$. The friction coefficient of seal ring contacts for top/bottom parts was $\mu=0.02$. Pressure load was applied at the left side of the ring. At the contacting surfaces where both pressure load and moving contact load could be applied simultaneously, 'pressure penetration' condition was applied. Bottom part was fixed. Top part moved down by $1.4 \mathrm{~mm}$ at the first step to give $20 \%$ squeeze, and pressure was applied at the second step. 


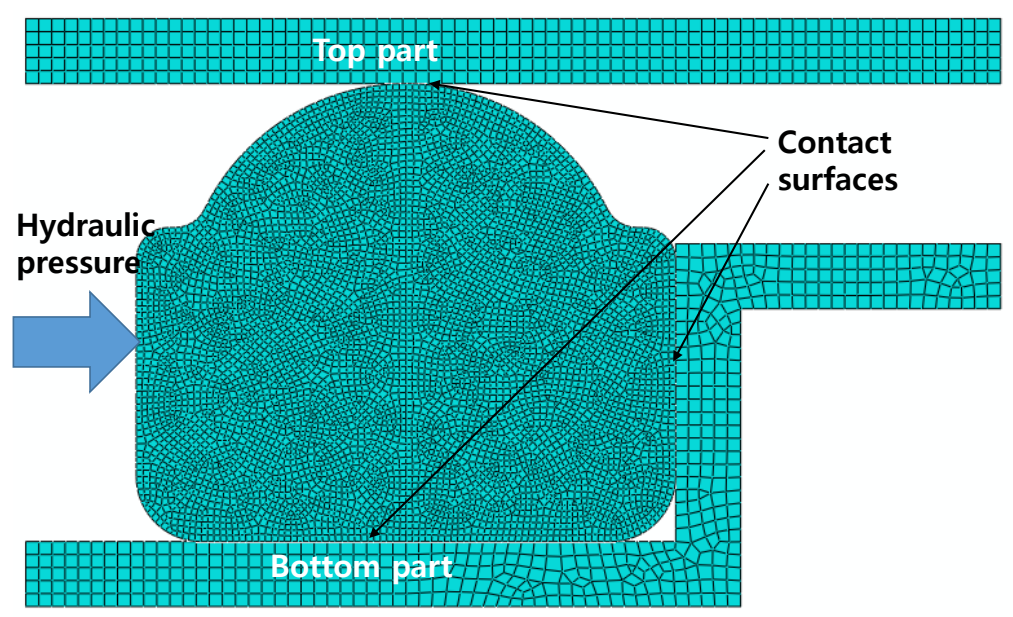

Fig. 12. Finite element model of the sealing system

Top and bottom parts are steel, and material properties were taken as E=206 GPa and Poisson's ratio v=0.32. D-ring was made from araldite based material. Therefore, time and temperature dependent properties were measured by dynamic mechanical analyzer according to ASTM D4065 using DMA analyzer (Q800, TA Instruments). Rectangular samples measuring $60 \mathrm{~mm}$ x $12 \mathrm{~mm}$ x $3 \mathrm{~mm}$ were prepared from a mixture of Araldite B41 and hardener HT 903 of which manufacturing conditions were the same as D-ring specimen. Three point bending test was done with $1.0 \mathrm{~Hz}$ frequency in the temperature range from $25^{\circ} \mathrm{C}$ to $150^{\circ} \mathrm{C}$. The results from the DMA test are shown in Fig. 13. It is shown that the araldite used in this research had a glass transition temperature, $\mathrm{Tg}$ of $110^{\circ} \mathrm{C}$. At the stress freezing temperature of $120{ }^{\circ} \mathrm{C}$, long term storage modulus was about $25 \mathrm{MPa}$.

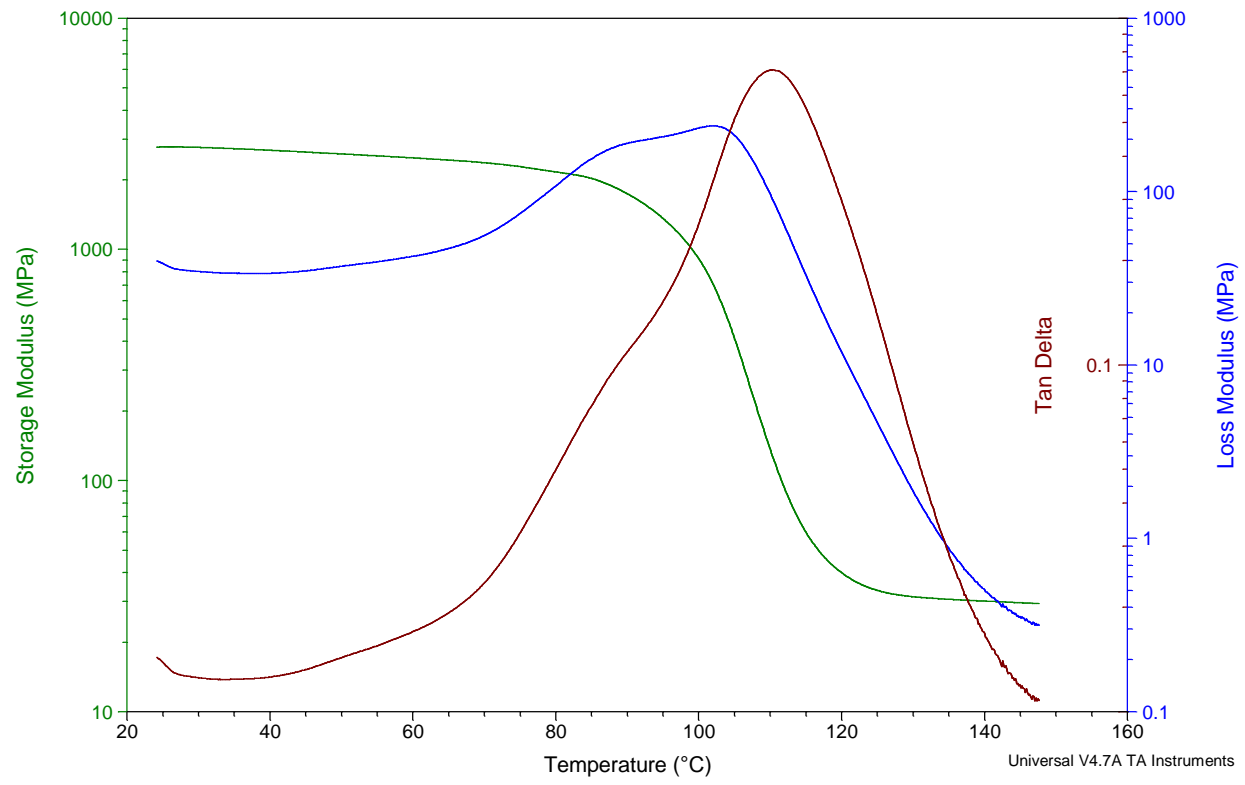

Fig. 13. Storage modulus, loss modulus, and $\tan \delta$ of epoxy specimen

Uniaxial tensile test was performed according to ASTM D638 at $120^{\circ} \mathrm{C}$ with loading rate of $1.0 \mathrm{~mm} / \mathrm{min}$. From the DMA test and unixial test results, hyperelastic material model of araldite was assumed. A simple Mooney-Rivlin strain energy function (strain energy potential order $=1$ ) in eq. (4) was used.

$$
W=C_{10}\left(I_{1}-3\right)+C_{01}\left(I_{2}-3\right)
$$

where $\boldsymbol{W}$ is the strain energy potential, $I_{l}$ and $I_{2}$ are the first and second invariants of the deviatoric strain, and $C_{10}$ and 
$C_{01}$ are Rivlin coefficients from experimental data. In this study, $C_{10}$ and $C_{01}$ were taken as 1.9 and 0.5 respectively.

The isochromatic fringes of the finite element (FE) model of $\mathrm{H}_{1} / \mathrm{H}_{2}=2.5$ under the hydraulic pressure of $0.98 \mathrm{MPa}$ is shown in Fig. 14 (a). The stress-optic law in eq. (2) was used to create fields. Note that the unit of the contour is fringe $\operatorname{order}(\mathrm{N})$, and the number of field in the legend is a dummy number. From the figure, under combined squeeze and hydraulic pressure, the seal is forced against the front side of the groove, resulting in deformation of seal to form 3 sealing surfaces similar to those in the actual experimental set up. The areas of contact between the seal and the mating surfaces serve as barriers preventing leakages. It is evident from the FE model that the as fluid pressure increase, the seal material is forced into the region near the extrusion gap in a way similar to experimental observation. A comparison of the isochromatics from FE model indicates remarkable similarity with the actual photoelastic ones in Fig. 4. The isochromatics on the pressure side are almost vertical just like in the case of experimental fringes. The overlay showing the deformation of the seals at incremental pressures are shown in Fig 14(b). The deformations from numerical model are very similar to those obtained from experiment. As a result of the remarkable similarities, the numerical model developed in this study can be effectively used to predict the behaviour of the seal during the normal operational conditions.

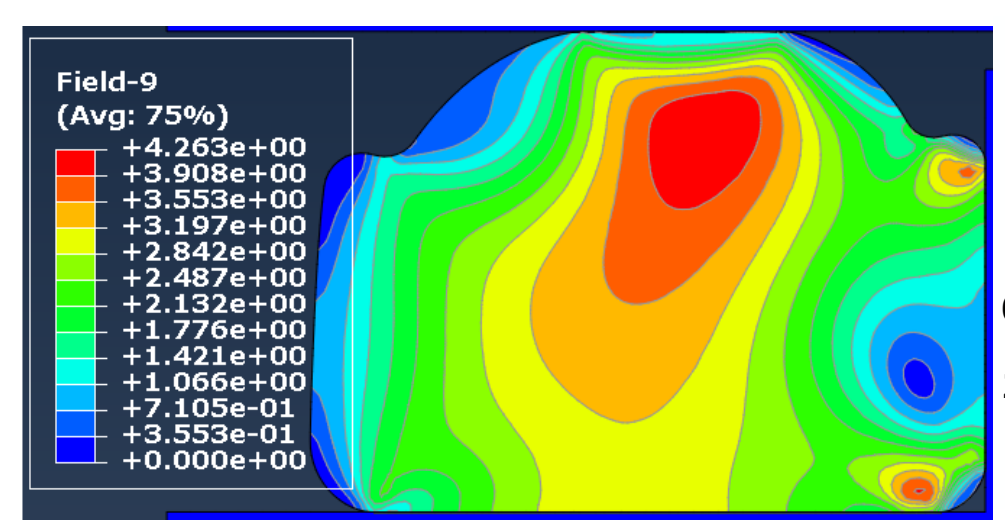

(a) $0.98 \mathrm{MPa}, \mathrm{H}_{1} / \mathrm{H}_{2}=2.5$

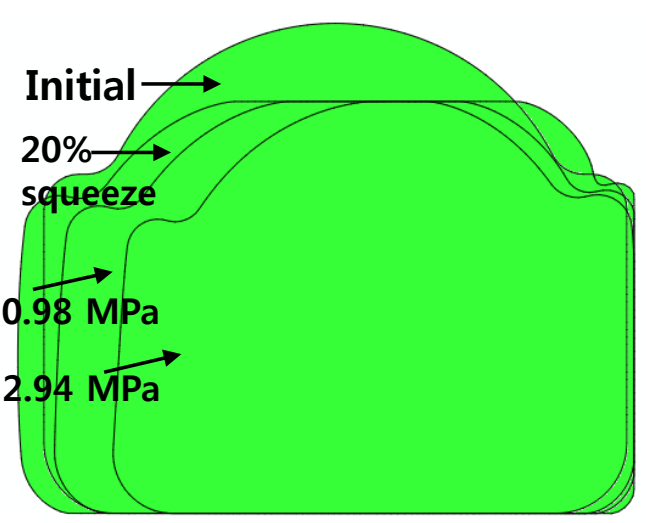

(b) Overlay, $\mathrm{H}_{1} / \mathrm{H}_{2}=2.5$

Fig.14. Results of finite element analysis of $\mathrm{H}_{1} / \mathrm{H}_{2}=2.5$ under the hydraulic pressure of 0.98 MPa. (a) Numerical isochromatic fringes and (b) overlay of deformed shape as a function of time increment

\section{Conclusion}

In this paper, performance behaviour of seals during operation were investigated in order to contribute to better designs of seals and seal systems commonly used in aircraft and rocket propulsion parts. Both experimental and numerical methods have been used. Experimental work is particularly useful not only for direct application of results in industry but also as a powerful tool to verify theoretical and numerical predictions. It was found that significant improvements in mechanical seal performance can be achieved by carefully combining the rectangular and circular profiles into one seal, called the D-shaped seal. The folding mechanism at the step of the seal with $\mathrm{H}_{1} / \mathrm{H}_{2}=2.5$ was found to contribute the seal's best performance. It was also found that for each loading condition, the contact stresses were greater than the applied medium pressure. This important sealing criterion confirmed that the new seal geometry has good sealing ability. Contrary to previous studies, contact stresses were found to possess a non Hertzian profile; and as medium pressure increased, stresses at the contact surface became almost uniform. By measuring the contact lengths using a video microscope, the seal with the new geometric condition recorded higher contact lengths compared with seals with circular profile. The numerical fringe patterns and deformation behavior from FEM showed remarkable similarity when compared with the photoelastic experiment results. From this study, it is shown that the seal with the new cross sectional profile can offer new possibilities as novel leak tight seal for high pressure applications.

\section{Acknowledgement}

(1) This work was supported by the 2016 Yeungnam University Research Grant. 
Mose, Dong-Kil Shin, Dong-Chul Shin,

(2) This research was supported by Basic Science Research Program through the National Research Foundation of Korea (NRF) funded by the Ministry of Education (NRF-2015R1D1A1A01061205).

\section{References}

Bignardi C, Manuello Bertetto A. and Mazza L., Photoelastic Measurements and Computation of Stress Field and Contact Pressure in a Pneumatic Lip Seal, Tribology International, Vol. 32, (1999), pp. 1-13.

Green Itzhak and English Capel, Stresses and deformation of compressed elastomeric O-ring seals, 14th International Conference on Fluid Sealing, Firenze, Italy, (6-8 April 1994), Organized by BHR Group Limited, Cranfield, Bedford, MK43 0AJ, UK

Hawong J. S., Nam J. H., Han S. L., Kwon O. S. and Park S. H., "A study on the analysis of O-ring under uniform squeeze rate and internal pressure by photoelastic experimental hybrid method", Journal of Mechanical Science and Technology, Vol. 23, (2009), pp. 2330-2340.

Hawong J. S., Nam J. H., Kim H. K., Liu Y, Kwon O. S. and Park H. S., A study on the development of a loading device using a photoelastic stress freezing method for the analysis of O-ring stress, Journal of Mechanical Science and Technology, Vol. 24, No. 3, (2010), pp. 693-701.

Hawong J.S, Shin D.C, Han S.L., A study on the development of reflective photoelastic experimental hybrid method on the static plane problems of orthotropic material, Key Engineering Materials, Vol. 324-325, (2006), pp. 1197-1200.

Johannesson H., Calculation of the pressure distribution in an O-ring seal contact, In: Proceedings of the 5th Leeds-Lyon symposium, (1978), pp. 379-87.

Johannesson, H.L. and Kassfeldt, E., Calculation of the pressure distribution in an arbitrary elastomeric seal contact, Wear, Vol. 130, (1989), pp. 3-15.

Karaszkiewicz A. Geometry and contact pressure of an O-ring mounted in a seal groove, Ind Eng Chem Res, Vol. 29, (1990), pp. 2134-2137.

Kim H.K, Park S.H, Lee H.G, Kim D.R, Lee Y.H. Approximation of contact stress for a compressed and laterally one side restrained O-ring, Engineering Failure Analysis, Vol. 14, (2007), pp. 1680-1692.

Kim Hyung-Kyu, Nam Jeong-Hwan, Hawong Jai-Sug and Lee Young-Ho, Evaluation of O-ring stresses subjected to vertical and one side lateral pressure by theoretical approximation comparing with photoelastic experimental results, Engineering Failure Analysis, Vol. 16, (2009), pp. 1876-1882.

Lee H. -S., Lee Y. -S., Chun B. -S., Kim S.-Y. and Baek J. -H., Contact stress analysis on the X-shape X-ring, Materialwissenschaft und Werkstofftechnik, Vol. 39, No. 2, (2008), pp. 193- 197.

Lin S. T. and Rowlands R. E., Hybrid stress analysis, Optics and Laser in Engineering, Vol. 32, (1999), pp. $257-298$.

Medri G. and Strozzi A., Mechanical Analysis of Elastomeric Seals by Numerical Methods, Ind. Eng. Chem. Prod. Res. Dev., Vol. 23, (1984), pp. 596-600.

Nam Jeong-Hwan, Hawong Jai-Sug, Han Song-Ling and Park Sung-Han, Contact Stress of O-ring under Uniform Squeeze Rate by Photoelastic Experimental Hybrid Method, Journal of Mechanical Science and Technology, Vol. 22, (2008), pp. 2337- 2349.

Nam Jeong-Hwan, Hawong Jai-Sug, Shin Dong-Chul and Mose Bruno R., A Study on the Behaviours and Stresses of Oring under Uniform Squeeze Rates and Internal Pressure by Transparent Type Photoelastic Experiment, Journal of Mechanical Science and Technology, Vol. 25, No. 9, (2011), pp. 2427-2438.

Parker, Extruded and Machined D-Ring Seals, Parker Hannifin Corporation, (2008).

Shin Dong-Chul, Hawong Jai-Sug, Lee Si-Wang, Alunda Ouma Bernard and Lim Hyun-Seok, Contact behavior analysis of X-ring under internal pressure and uniform squeeze rate using photoelastic experimental hybrid method, Journal of Mechanical Science and Technology, Vol. 28, No. 10, (2014), pp. 4063- 4073.

Strozzi, A. Static stresses in an unpressurized, rounded, rectangular, elastomeric seal. ASLE Trans., Vol. 29 No. 4, (1986), pp. 558-564.

White C. M. and Denny D. F., The sealing mechanism of flexible packings, Ministry of Supply, Scientific and Technical Memorandum 3/47, (1947), (Reprinted in 1972, BHRA Fluid Engineering, Cranfield, Bedfordshire). 